УДК 616.895.8:615.03:577.171

Для цитирования: Горобец Л.Н., Буланов В.С., Литвинов А.В. Сравнительная динамика уровней пролактина, эстрадиола и тестостерона у пациенток с параноидной шизофренией в процессе антипсихотической терапии. Сибирский вестник психиатрии и наркологии. 2018; 2 (99): 127-132. https://doi.org/10.26617/1810-3111-2018-2(99)-127-132

\title{
Сравнительная динамика уровней пролактина, эстрадиола и тестостерона у пациенток с параноидной шизофренией в процессе антипсихотической терапии
}

\author{
Горобец Л.Н., Буланов В.С., Литвинов А.В.
}

Московский НИИ психиатрии - филиал НМИЦПН им. В.П. Сербского

Россия,107258, Москва, ул. Потешная, 3

\section{PEЗЮME}

Противоречивость данных литературы относительно влияния антипсихотиков первого и второго поколений на уровни пролактина и половых гормонов диктует необходимость проведения сравнительных исследований уровней указанных гормонов на разных этапах антипсихотической терапии. Цель: изучение особенностей динамики уровней пролактина, эстрадиола и тестостерона в сыворотке крови у пациенток с параноидной шизофренией в процессе антипсихотической терапии. Материалы и методы. У 131 пациентки с параноидной шизофренией в процессе монотерапии оланзапином, клозапином, кветиапином и галоперидолом исследовались показатели пролактина, эстрадиола и тестостерона иммуноферментным методом. Гормональные показатели определяли до начала терапии (фон), через 3-4 и 6-8 недель терапии. Результаты. Терапия галоперидолом независимо от фоновых значений приводит к значимому (p<0,01) повышению содержания пролактина в крови, а терапия клозапином и кветиапином - к значимому (p<0,01) снижению изначально повышенного уровня пролактина. Характер влияния оланзапина зависит от фонового уровня пролактина: при уровне пролактина более 1000 мМЕ/л препарат приводит к снижению уровня гормона, менее $1000 \mathrm{MME/л} \mathrm{-} \mathrm{повышает} \mathrm{его} \mathrm{и} \mathrm{не} \mathrm{изменяет}$ показателей пролактина, если они находятся в нормативном диапазоне. При терапии исследованными антипсихотиками отмечалось значимое $(\mathrm{p}<0,01)$ снижение уровней эстрадиола на фоне значимого $(\mathrm{p}<0,01)$ повышения уровней тестостерона. Выводы Авторами обосновывается гипотеза о том, что снижение уровня эстрадиола на фоне повышения уровня тестостерона у пациенток с параноидной шизофренией способствует развитию у них менструальных дисфункций. Ключевую роль в повышении уровня тестостерона у женщин в процессе антипсихотической терапии играет снижение уровня эстрадиола и в меньшей степени изменение уровня пролактина.

Ключевые слова: шизофрения, антипсихотическая терапия, пролактин, эстрадиол, тестостерон.

\section{ВВЕДЕНИЕ}

В отечественной и зарубежной литературе последних десятилетий большое внимание уделяется исследованиям особенностей изменений уровня пролактина у больных шизофренией при антипсихотической терапии. В большинстве работ приводятся данные о значительном влиянии рисперидона и галоперидола на развитие гиперпролактинемии $[1,2$, $3,4,5]$. В ряде других исследований терапия такими антипсихотиками второго поколения, как оланзапин, клозапин и кветиапин, характеризуется отсутствием заметного влияния на секрецию пролактина и, как следствие, отсутствием заметного влияния на развитие нарушений менструального цикла (НМЦ) $[6,7]$. Вместе с тем данные о влиянии АВП на секрецию периферических гормонов гипоталамогипофизарно-гонадной (ГГГ) оси, таких как эстрадиол и тестостерон, а также о возможных взаимовлияниях изменения секреции пролактина и указанных гормонов и формировании на этом фоне менструальных дисфункций весьма малочисленны и противоречивы $[8,9,10,11]$.

Так, в исследовании Т.J. Huber, M. Borsutzky, проводимом у пациентов, госпитализированных с острой психотической симптоматикой, не были обнаружены корреляции между уровнем пролактина и эстрадиола и различия между уровнем эстрадиола у пациентов как на терапии антипсихотиками, так и после ее прекращения [12]. Другие исследования пациентов с шизофренией обнаружили отрицательную корреляцию между пролактином и уровнем эстрадиола $[13,14,15]$. Интересно, что в указанных исследованиях описывалось снижение уровня эстрадиола и формирование гипогонадизма у женщин с шизофренией, однако данные о связях этих изменений с формированием НМЦ не были обнаружены.

В то же время в исследованиях, проводимых в области гинекологии и эндокринологии, приводятся данные о том, что гиперпролактинемия, а также изменение уровней половых гормонов (снижение эстрадиола и повышение тестостерона), особенно изменение их соотношения, приводит к развитию указанных нарушений $[16,17]$.

Цель - изучение особенностей динамики уровней пролактина, эстрадиола и тестостерона в сыворотке крови у пациенток с параноидной шизофренией в процессе антипсихотической терапии.

\section{МАТЕРИАЛ И МЕТОДЫ}

Материалом для исследования послужили данные обследования 131 пациентки в возрасте от 18 до

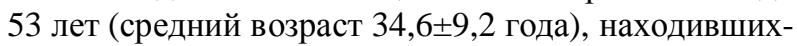
ся на стационарном лечении в клиниках МНИИП филиала ФГБУ «НМИЦПН им. В.П. Сербского» Минздрава России и отделениях ГКП № 4 им. П.Б. Ганнушкина. 
У всех обследованных пациенток была диагностирована параноидная шизофрения (F20.0) в соответствии с критериями МКБ-10.

Критерии включения в исследование: возраст от 18 до 53 лет; отсутствие органических заболеваний ЦНС; отсутствие эндокринных заболеваний; отсутствие тяжелых форм соматических и гинекологических заболеваний; отсутствие беременности и лактации; «wash-out»-период в течение 7-10 дней перед назначением антипсихотических препаратов в тех случаях, когда больные, длительно страдающие шизофренией, до начала исследования получали антипсихотическую терапию. От всех обследованных пациентов было получено информированное согласие на участие в исследовании.

Основную группу составили 106 пациенток (27,36 \pm 7,2 года), которым проводилась монотерапия одним из антипсихотиков второго поколения (АВП). Отбор больных в подгруппы осуществлялся рандомизированным способом. Таким образом, в зависимости от назначаемого препарата было сформировано 3 основные подгруппы больных (I - при терапии клозапином, II - оланзапином, III - кветиапином). В качестве подгруппы сравнения обследовано 25 женщин, получавших терапию антипсихотиком первого поколения (АПП) - галоперидолом. Сравниваемые выборки были сопоставимы по основным клинико-демографическим показателям (табл. 1).

Т а б ли ц а

Клинико-демографические характеристики исследованных больных

\begin{tabular}{|c|c|c|c|c|}
\hline \multirow{2}{*}{ Показатель } & \multicolumn{3}{|c|}{ Основная группа } & \multirow{2}{*}{\begin{tabular}{|c|} 
Группа \\
сравнения \\
Галоперидол \\
(IV групп) \\
\end{tabular}} \\
\hline & $\begin{array}{l}\text { Клозапин } \\
\text { (І группа) }\end{array}$ & $\begin{array}{l}\text { Оланзапин } \\
\text { (II группа) }\end{array}$ & \begin{tabular}{|l} 
Кветиапин \\
(III группа)
\end{tabular} & \\
\hline Количество & $35(26,7 \%)$ & $37(28,3 \%)$ & $34(26 \%)$ & $25(19 \%)$ \\
\hline $\begin{array}{l}\text { Средний воз- } \\
\text { раст }(\mathrm{M} \pm \mathrm{s})\end{array}$ & $36,7 \pm 7,4$ & $32,5 \pm 10$ & $35,2 \pm 9,7$ & $34,2 \pm 5,1$ \\
\hline $\mathrm{F} 20.00$ & $100 \%$ & $100 \%$ & $100 \%$ & $100 \%$ \\
\hline $\begin{array}{l}\text { Длительность } \\
\text { заболевания, } \\
\text { лет }(\mathrm{M} \pm \mathrm{s}) \\
\end{array}$ & $9,3 \pm 5,2$ & $8,4 \pm 4,7$ & $7,6 \pm 4,5$ & $11,2 \pm 5,8$ \\
\hline $\begin{array}{l}\text { Средняя сут. } \\
\text { доза, мг }(\mathrm{M} \pm \mathrm{s})\end{array}$ & $343,4 \pm 91$ & $15,5 \pm 5,6$ & $422 \pm 43$ & $25,3 \pm 10,5$ \\
\hline НМЦ & $51 \%$ & $51 \%$ & $44 \%$ & $52 \%$ \\
\hline
\end{tabular}

Исследование пациентов проводилось динамически на определенных этапах терапевтического процесса: фон (1-й этап) - до назначения терапии; 3-4-я неделя терапии (2-я этап) и 6-8-я неделя терапии (3й этап).

Основными методами работы являлись: клинико-психопатологический, клинико-эндокринологический, биохимический и клинико-статистический.

Определение содержания гормонов пролактина, эстрадиола и тестостерона проводилось натощак в утренние часы в сыворотке крови иммуноферментным методом на фотометре вертикального сканирования Multiscan Agent («Labsystems», Финляндия) с использованием реактивов фирмы «АлкорБио». Референсные значения гормонов: пролактин 70-700 мМЕ/л; эстрадиол - 30-120 пг/мл; тестостерон - 0,2-4,0 нмоль/л.
Статистическая обработка результатов осуществлялась с использованием компьютерной статистической программы «Statistica» версия 7.0 (StatSoft, Inc., USA для Windows) с вычислением средних значений $(\mathrm{M})$ и ошибки среднего $( \pm \mathrm{SE})$. Для сравнения показателей применялись непараметрические методы статистической оценки: для сравнения двух зависимых переменных - критерий Вилкоксона, анализ межгрупповых различий проводился с помощью теста Манна-Уитни. Для исследования взаимосвязи между переменными использовался ранговый коэффициент корреляции Спирмена (r). Различия считались достоверными при р<0,01.

\section{РЕЗУЛЬТАТЫ}

Фоновые уровни пролактина превышали нормативные показатели в среднем на $80 \%(\mathrm{p}<0,01)$ как в основных подгруппах, так и в подгруппе сравнения. При этом фоновые показатели во всех подгруппах не имели статистически значимых различий (табл. 2). В процессе терапии клозапином и кветиапином происходила нормализация уровней пролактина уже на 2-м этапе исследования вне зависимости от величин фоновых показателей.

Т а б ли ц а 2

Динамика уровня пролактина у пациенток с параноидной шизофренией в процессе антипсихотической терапии

\begin{tabular}{l|l|l|l|l} 
Этап & $\begin{array}{c}\text { Клозапин } \\
\text { (I группа, }\end{array}$ & $\begin{array}{l}\text { Оланзапин } \\
\text { (II группа, }\end{array}$ & $\begin{array}{c}\text { Кветиапин } \\
\text { (III группа, }\end{array}$ & $\begin{array}{c}\text { Галоперидол } \\
\text { (IV группа, }\end{array}$ \\
\hline
\end{tabular} \begin{tabular}{c|c|c|c}
$\begin{array}{c}\text { (I группа, } \\
\mathrm{n}=29)\end{array}$ & $\begin{array}{c}\text { (II группа, } \\
\mathrm{n}=46)\end{array}$ & $\begin{array}{c}\text { (III группа, } \\
\mathrm{n}=32\end{array}$ & $\left.\begin{array}{c}\text { (IV групп } \\
\mathrm{n}=33\end{array}\right)$ \\
\hline
\end{tabular}

\begin{tabular}{|l|l|l|l|l|}
\hline 1 -й этап & $1450,2 \pm 260,2$ & $1132,0 \pm 162,2$ & $1676,8 \pm 237,5$ & $1564,4 \pm 139$ \\
\hline 2 -й
\end{tabular} \begin{tabular}{l|l|l|l|l|} 
2-й этап & $447,5 \pm 54,5 *$ & $799,4 \pm 79,8 *$ & $648,8 \pm 188,1 *$ & $1892,0 \pm 141^{*}$ \\
\hline
\end{tabular} \begin{tabular}{lll|l|l|l|}
\hline 3-й этап & $383,3 \pm 46,6^{*}$ & $696,6 \pm 76,2^{*}+$ & $525,2 \pm 137,3 *$ & $1843,5 \pm 126^{*}$ \\
\hline
\end{tabular}

П р и м е ч а н и е. Значимость внутригрупповых различий по сравнению с фоновыми значениями: * - p<0,01 (критерий Вилкоксона); значимость внутригрупповых различий между 2-м и 3-м этапами: $\dagger-$ p $<0,01$ (критерий Вилкоксона).

Характер влияния оланзапина зависел от фонового уровня пролактина в сыворотке крови: при исходно нормативных уровнях пролактина оланзапин не оказывал своего пролактогенного действия (12\% случаев). При цифрах, превышающих 1000 мМЕ/л, происходило снижение и нормализация уровня гормона к 6-8-й неделе терапии (70\% случаев). У пациенток с уровнем пролактина в пределах 700-1000 $\mathrm{MME/л} \mathrm{происходило} \mathrm{повышение} \mathrm{его} \mathrm{содержания}$ через месяц терапии с последующим его снижением и в ряде случаев с нормализацией к 6-8-й неделе лечения (18\% случаев). Таким образом, средние показатели пролактина в целом по подгруппе не превышали референтные значения лишь на 3-м этапе исследования. Терапия галоперидолом сопровождалась статистически значимым ( $\mathrm{p}=0,00005)$ подъемом уровня пролактина в среднем на $90 \%$ от фоновых показателей уже на 3-4-й неделе и незначительным его снижением в конце терапии ( $\mathrm{p}=0,00002$ относительно фона). Пролактогенное влияние галоперидола не зависело от фоновых показателей пролактина.

На 1-м этапе (фон) исследования периферических гормонов ГГГ-оси было обнаружено, что у части пациенток уровень эстрадиола находился ниже нормативных показателей в среднем на $12 \%$. 
Т а б л и ц а 3 Динамика уровня эстрадиола у пациенток с параноидной шизофренией в процессе антипсихотической терапии (N ФФ 30-120 пг/мл)

\begin{tabular}{|c|c|c|c|c|}
\hline Этап & $\begin{array}{c}\text { Клозапин } \\
\text { (I групп, } \\
\mathrm{n}=29)\end{array}$ & $\begin{array}{c}\text { Оланзапин } \\
\text { (II группа, } \\
\mathrm{n}=46 \text { ) }\end{array}$ & $\begin{array}{c}\text { Кветиапин } \\
\text { (III группа, } \\
\mathrm{n}=32 \text { ) }\end{array}$ & $\begin{array}{c}\text { Галоперидол } \\
\text { (IV группа, } \\
\mathrm{n}=33 \text { ) }\end{array}$ \\
\hline 1 -й этап & $57,1 \pm 6,2$ & $71,2 \pm 8,9$ & $65,3 \pm 7,8$ & $65,3 \pm 8,7$ \\
\hline 2-й этап & $29,7 \pm 5,6^{*}$ & $35,8 \pm 6,7^{*}$ & $34,9 \pm 7,8^{*}$ & $37,8 \pm 9,7 \dagger$ \\
\hline 3-й этап & $26,3 \pm 7,2^{*}$ & $36,7 \pm 8,7^{*}$ & $36,4 \pm 9,1^{*}$ & $32,7 \pm 6,4^{*}$ \\
\hline
\end{tabular}

П р и м е ч а н и е. Значимость внутригрупповых различий по сравнению с фоновыми значениями на выделенных этапах терапии: * - p<0,01; $\uparrow-$ p $<0,001$ (критерий Вилкоксона).

По частоте встречаемости пациенток с уровнем эстрадиола ниже референсных показателей до включения в исследование подгруппы не различались между собой и находились в пределах от 42,8\% до 53\% случаев. Обнаружено, что в I и II подгруппах низкие фоновые показатели эстрадиола коррелировали с высокими фоновыми значениями пролактина $(\mathrm{r}=-0,48 ; \mathrm{p}=0,01$ и $\mathrm{r}=-0,67$, при $\mathrm{p}=0,01$ соответственно подгруппам). В процессе исследования было выявлено дальнейшее снижение среднего уровня эстрадиола у пациенток во всех подгруппах, при этом данные изменения были выраженными и статистически значимыми уже на 3-4-й неделе исследования ( $<<0,01)$.

Количество пациенток с уровнем эстрадиола ниже нормативных показателей ко 2-му этапу терапии было сопоставимым в I, II и III подгруппах и находилось в пределах от $72,9 \%$ до $85,3 \%$ случаев. У больных в подгруппе сравнения на 2-м этапе исследования частота встречаемости пациенток с низкими значениями эстрадиола не превышала фоновых показателей $(44 \%)$ и значимо $(\mathrm{p}<0,01)$ возрастала (до 76\%) лишь к 6-8-й неделе исследования. В то время как при терапии галоперидолом и оланзапином выявлялась отрицательная корреляционная зависимость между снижением уровня эстрадиола и повышением уровня пролактина: по окончании терапии галоперидолом ( $\mathrm{r}=-0,59$, при $\mathrm{p}=0,03)$ и на 2м и 3-м этапах терапии оланзапином ( $\mathrm{r}=-0,58$, при $\mathrm{p}=0,03$ и $\mathrm{r}=-0,75$, при $\mathrm{p}=0,002$ соответственно этапам).

В отличие от эстрадиола, фоновые уровни тестостерона у всех пациенток находились в нормативном диапазоне. Динамика уровня тестостерона в процессе исследования имела противоположную тенденцию по отношению к уровню эстрадиола (табл. 4).

Т а б л и ц а 4 Динамика уровня тестостерона у женщин с параноидной шизофренией в процессе антипсихотической терапии

\begin{tabular}{|c|c|c|c|c|}
\hline Этап & $\begin{array}{c}\text { Клозапин } \\
\text { (I групп, } \\
\text { n=29) }\end{array}$ & $\begin{array}{c}\text { Оланзапин } \\
\text { (II группа, } \\
\text { n=46) }\end{array}$ & $\begin{array}{c}\text { Кветиапин } \\
\text { (III группа, } \\
\text { n=32) }\end{array}$ & $\begin{array}{c}\text { Галоперидол } \\
\text { (IV группа, } \\
\text { n=33) }\end{array}$ \\
\hline 1 -й этап & $1,9 \pm 019$ & $2,6 \pm 0,22$ & $2,6 \pm 0,28$ & $2,4 \pm 0,2$ \\
\hline 2-й этап & $2,2 \pm 0,22$ & $2,8 \pm 0,24$ & $2,7 \pm 0,32$ & $3,0 \pm 0,2 \dagger$ \\
\hline 3-й этап & $2,6 \pm 0,21 \dagger$ & $3,2 \pm 0,22^{*}$ & $3,4 \pm 0,19^{*}$ & $3,4 \pm 0,1 \dagger$ \\
\hline
\end{tabular}

П р и м е ч а н и е. Значимость внутригрупповых различий по сравнению с фоновыми значениями на выделенных этапах терапии: * ${ }^{*}$ p $<0,01 ; \dagger-\mathrm{p}<0,001$ (критерий Вилкоксона).
При терапии АВП (клозапин, оланзапин и кветиапин) отмечалось статистически значимое $(\mathrm{p}<0,01)$ повышение уровня тестостерона на 6-8-й неделе терапии, а в подгруппе галоперидола - уже на 3-4-й неделе исследования. Число пациенток с повышением уровня тестостерона в среднем на $25 \%$ при терапии всеми исследуемыми препаратами составляло ко 2-му этапу от $59 \%$ до 74,2\%. К 6-8-й неделе терапии повышение уровней тестостерона выше референсных показателей наблюдалось в диапазоне от $17,6 \%$ до $26,0 \%$ случаев и встречалось с одинаковой частотой во всех подгруппах. В ходе исследования было обнаружено, что низкие уровни эстрадиола коррелировали с высокими значениями тестостерона на 3-м этапе терапии как АВП, так и галоперидолом $(\mathrm{r}=-0,57, \mathrm{p}=0,002-$ терапия с применением оланзапина; $\mathrm{r}=-0,56, \mathrm{p}=0,001$ - терапия клозапином; $\mathrm{r}=-0,47, \mathrm{p}=0,003$ - терапия кветиапином; $\mathrm{r}=-0,81, \mathrm{p}=0,001$ - терапия галоперидолом).

\section{ОБСУЖДЕНИЕ}

На современном этапе развития психофармакотерапии проблема повышения уровня пролактина при терапии антипсихотиками (АПП и АВП) у больных шизофренией является крайне актуальной и наиболее часто обсуждаемой. Однако большинство авторов ограничивается лишь констатацией того факта, что антипсихотические препараты оказывают различное влияние на уровень пролактина в крови в зависимости от специфики их аффинитета к дофаминовым рецепторам в тубероинфундибулярной области головного мозга, дозы препарата, а также в ряде случаев от половой принадлежности пациента [14, 15, 18].

Результаты, полученные в нашем исследовании, подтверждают данные научной литературы о том, что терапия галоперидолом, вне зависимости от фоновых значений, приводит к повышению содержания пролактина в крови. Пролактогенный эффект галоперидола связан с тем обстоятельством, что препарат оказывает выраженное селективное блокирующее действие на $\mathrm{D}_{2}$-рецепторы тубероинфундибулярной области головного мозга, к которой относятся гипоталамус и гипофиз [19]. Клозапин и кветиапин недифференцированно блокируют дофаминовые рецепторы [11] и не оказывают тем самым влияния на секрецию пролактина, что подтверждается результатами нашего исследования и выражается в значимом $(\mathrm{p}<0,01)$ снижении изначально повышенного уровня пролактина у больных в процессе терапии указанными препаратами. Вместе с тем обращает на себя внимание неоднозначность пролактогенного эффекта оланзапина, что находит свое подтверждение и в ряде исследований [20, 21]. По всей вероятности, это связано с тем, что по выраженности влияния на $\mathrm{D}_{2}$-рецепторы оланзапин ближе к галоперидолу, несмотря на то что по классификации [2] относится к поливалентным антипсихотикам и по влиянию на ряд других рецепторов сходен с клозапином и кветиапином, относящимся к той же группе препаратов. 
Следует также отметить, что обнаруженные нами высокие (превышающие нормативные показатели) фоновые уровни пролактина у пациенток во всех подгруппах, несмотря на проводимый «washout» период перед началом исследования, могут свидетельствовать о влиянии предшествующей психофармакотерапии на секрецию пролактина, так как все исследуемые больные в анамнезе неоднократно принимали различные антипсихотические препараты.

Полученные нами данные в отношении содержания эстрадиола в сыворотке крови на 1-м этапе исследования согласуются с результатами, приводимыми рядом авторов [7, 15, 22], в публикациях которых приводятся данные о снижении уровня эстрадиола у пациенток в острой фазе шизофренического процесса. В то же время мы полагаем, что низкие (ниже референсных значений) уровни эстрадиола, обнаруженные нами у больных на 1-м этапе (фон), были связаны в первую очередь с высокими (превышающими нормативные) показателями уровнями пролактина у этих больных до включения в исследование, а не с обострением психопатологической симптоматики. Наше предположение, в частности, подтверждается наличием отрицательных корреляционных связей между уровнями пролактина и эстрадиола у больных, обнаруженным до начала терапии. Дальнейшее снижение уровня эстрадиола на фоне повышения уровня пролактина у больных в процессе терапии оланзапином и галоперидолом, а также наличие отрицательных корреляций между указанными гормонами полностью подтверждает гипотезу о влиянии гиперпролактинемии любого генеза на снижение секреции эстрадиола как на центральном (гипофиз) - (влияние на секрецию ЛГ и $\Phi С)$, так и на периферическом (яичники) - (влияние на секрецию эстрадиола и тестостерона) уровнях, приводимую в работах отечественных и зарубежных исследователей [3, 17].

Как известно, снижение уровня эстрадиола у женщин при гинекологической и эндокринной патологии приводит к НМЦ. Помимо того, в развитии менструальных дисфункций определенную роль играют изменения уровней и других гормонов гонадной оси, в частности тестостерона. Наши исследования подтвердили это предположение в отношении нейролептических НМЦ. Было выявлено, что динамика уровня тестостерона имела противоположную направленность по отношению к изменениям эстрадиола. Было также показано, что для терапии клозапином, оланзапином и кветиапином характерно статистически значимое $(\mathrm{p}<0,01)$ повышение уровня тестостерона к 6-8-й неделе, а галоперидолом - уже к 3-4-й неделе. Обращает на себя внимание тот факт, что в отличие от эстрадиола фоновые уровни тестостерона у всех пациенток находились в нормативном диапазоне, и лишь к 3-4-й неделе резко возрастало число пациенток со значимым ( $<0,01)$ повышением уровня тестостерона при терапии всеми исследуемыми препаратами.
Тем не менее лишь к 6-8-й неделе у части пациенток уровень тестостерона превысил нормативные показатели. Кроме того, на 3-м этапе исследования было выявлено изменение соотношения эстрадиола и тестостерона в среднем в пределах 11/1, которое в норме составляет 15/1 и, как предполагается [23], может служить одним из диагностических критериев дисбаланса, приводящего к развитию НМЦ, которые отмечались у исследованных пациенток.

Исходя из полученных данных, можно высказать предположение о том, что в повышении уровня тестостерона ключевую роль играет снижение уровня эстрадиола, в меньшей степени - изменение уровня пролактина. Это предположение подтверждается и тем фактом, что низкие уровни эстрадиола коррелируют с высокими значениями тестостерона на всех этапах терапии как АВП, так и галоперидолом, тогда как корреляционные взаимосвязи между уровнями пролактина и тестостерона более слабые и выявляются не на всех этапах терапии.

Эта тенденция, характеризующая терапию оланзапином и галоперидолом, наряду с обнаруженной в начале исследования корреляцией низких значений эстрадиола и высоких показателей пролактина, свидетельствует о том, что нейролептическая гиперпролактинемия непосредственно влияет на синтез эстрадиола в яичниках. По литературным данным [24], пролактин, конкурентно связываясь с рецепторами гонадотропинов на уровне яичников, тормозит влияние гонадотропинов на стероидогенез и снижает чувствительность яичников к экзогенным и эндогенным гонадотропинам. Кроме того, в экспериментальных исследованиях доказано, что пролактин при добавлении к культуре гранулезных клеток преовуляторных фолликулов оказывает ингибирующее действие на синтез эстрадиола и не влияет на синтез тестостерона [23].

\section{ВЫВОДЫ}

1. Средний фоновый уровень пролактина у исследованных пациенток превышал нормативные показатели, что, по всей вероятности, непосредственно связано с предшествующей антипсихотической терапией.

2. Галоперидол значимо $(\mathrm{p}<0,01)$ повышал уровень пролактина, а клозапин и кветиапин значимо $(\mathrm{p}<0,01)$ снижали изначально повышенные уровни гормона. Влияние оланзапина на показатели пролактина зависели от его фоновых величин.

3. При терапии изученными препаратами отмечалось нарушение соотношения эстрадиола и тестостерона, пусковым механизмом которого является гиперпролактинемия.

4. Повышение уровней тестостерона в первую очередь связано со снижением секреции эстрадиола, а не с изменениями уровня пролактина.

5. Изменение соотношения эстрадиола и тестостерона, наряду с повышением уровня пролактина, может являться основой для развития НМЦ у пациенток с параноидной шизофренией при проведении антипсихотической терапии. 


\section{КОНФЛИКТ ИНТЕРЕСОВ}

Авторы заявляют об отсутствии конфликта интересов в связи с публикацией данной статьи.

\section{ИСТОЧНИК ФИНАНСИРОВАНИЯ}

Исследование не имело спонсорской поддержки. СООТВЕТСТВИЕ ПРИНЦИПАМ ЭТИКИ

Исследование проводилось в соответствии с Хельсинской декларацией об этических принципах проведения медицинских исследований у людей и заключением ЛЭК ФГУ «Московский НИИ психиатрии Росздрава» (протокол № 43/2 от 27 апреля 2009 г.).

\section{ЛИТЕРАТУРА/REFERENCES}

1. Madhusoodanan S., Parida S., Jimenez C. Hyperprolactinemia associated with psychotropics - a review. Hum. Psychopharmacol. Clin. Exp. 2010; 25: 281-297.

2. Haddad P.M., Wieck A. Antipsychotic-induced hyperprolactinaemia: mechanisms, clinical features and management. Drugs. 2004; 64; 20: 2291-2314.

3. Peuskens J., Pani L., Detraux J., De Hert M. The effects of novel and newly approved antipsychotics on serum prolactin levels: a comprehensive review. CNS Drugs. 2014; 28: 421-453.

4. Горобец Л.Н. Нейроэндокринные дисфункции и нейролептическая терапия. Москва: ИД «Медпрактика-М», 2007: 312. Gorobets L.N. Neyroendokrinnyie disfunktsii i neyrolepticheskaya terapiya [Neuroendocrine dysfunctions and neuroleptic therapy]. Moscow: Publishing House "Medpraktika-M", 2007: 312 (in Russian).

5. Корнетова Е.Г., Микилев Ф.Ф., Лобачева О.А., Бородюк Ю.Н., Бойко А.С., Семке А.В. Клинические и иммунологические особенности гиперпролактинемии у пациентов с шизофренией, принимающих рисперидон. Сочиальная и клиническая психиатрия. 2016; 26 (1): 5-11. Kornetova E.G., Mikilev F.F., Lobacheva O.A., Borodyuk Yu.N., Boyko A.S., Semke A.V. Klinicheskie i immunologicheskie osobennosti giperprolaktinemii u patsientov s shizofreniey, prinimayuschih risperidon [Clinical and immunological features of hyperprolactinemia in patients with schizophrenia receiving risperidone]. Sotsialnaya $i$ klinicheskaya psihiatriya - Social and Clinical Psychiatry. 2016; 26 (1): 5-11 (in Russian)

6. Perkins D.O. Prolactin- and Endocrine-Related Disorders in Schizophrenia / Medical Illness and Schizophrenia (Ed. by Meyer J.M. and Nasrallah H.A.). Amer. Psychiatr. Publ. Washington, London, 2003: 215-232.

7. Kulkarni J., Hayes E., Gavrilidis E. Hormones and schizophrenia. Curr Opin Psychiatry. 2012; Mar; 25 (2): 89-95. doi: 10.1097/YCO.0b013e328350360e.

8. Canuso C.M., Goldstein J.M., Wojcik J. et al. Antipsychotic medication, prolactin elevation, and ovarian function in women with schizophrenia and schizoaffective disorder. Psychiatry Res. 2002; 111; 1 (Aug 5): 11-20.

9. Lally J., MacCabe J.H. Antipsychotic medication in schizophrenia: a review. Br. Med. Bull. 2015; 114 (1): 169-179.
10. Cookson J., Hodgson R., Hiram J.W. Prolactin, hyperprolactinaemia and antipsychotic treatment: a review and lessons for treatment of early psychosis. J Psychopharmacol. 2012; 26: 42-51.

11. Egli M., Leeners B, Kruger T.H.C. Prolactin secretion patterns: basic mechanisms and clinical implications for reproduction. Reproduction. 2010; 140: 643-654.

12. Huber T.J., Borsutzky M., Schneider U., Emrich H.M. Psychotic disorders and gonadal function: evidence supporting the oestrogen hypothesis. Acta Psychiatr. Scand. 2004; 109; 4: 269-274.

13. Smith S., Wheeler M.J., Murray R., O'Keane V. The effects of antipsychotic - induced hyperprolactinaemia on the hypothalamic - pituitary - gonadal axis. Clin Psychopharmacol. 2002; 22; 2 (Apr): 109-114.

14. Bushe C., Shaw M., Peveler R. A review of the association between antipsychotic use and hyperprolactinaemia. J Psychopharmacol. 2008; 22: 46-55.

15. Riecher-Rössler A. Oestrogens, prolactin, hypothalamic - pituitary - gonadal axis, and schizophrenic psychoses. Lancet Psychiatry. 2017; Jan; 4 (1): 63-72. doi: 10.1016/S22150366(16)30379-0

16. Лавин Л. Эндокринология. М.: 1999: 1128. Lavin L. Endokrinologiya [Endocrinology]. Moscow, 1999: 1128 (in Russian).

17. Сметник В.П., Тумилович Л.Г. Неоперативная гинекология. Руководство для врачей. Книга 1. Изд 2-е. СПб., 1995: 223. Smetnik V.P., Tumilovich L.G. Neoperativnaya ginekologiya. Rukovodstvo dlya vrachey [Non-surgical gynecology. Handbook for physicians]. Book $1,2^{\text {nd }}$ edition. Saint-Petersburg, 1995: 223 (in Russian).

18. Kuepper R., Skinbjerg M., Abi-Dargham A. The dophamine dysfunction in schizophrenia revisited: new insights into topography and course. Handbook Experim. Pharmacol. 2012; 212: $1-26$.

19. Carlsson A., Waters N., Carlsson M.L. Neurotransmitter interactions in schizophrenia - therapeutic implications. Biol. Psychiatry. 1999; 46: 1388-1395.

20. Inder W.J., Castle D. Antipsychotic-induced hyperprolactinaemia. Aust N Z J Psychiatry. 2011; 45: 830-837.

21. Leucht S., Cipriani A., Spineli L. et al.. Comparative e-cacy and tolerability of 15 antipsychotic drugs in schizophrenia: a multiple treatments meta-analysis. Lancet. 2013; 382: 951-962.

22. Markham J.A. Sex steroids and schizophrenia. Rev. Endocr. Metab. Disord. 2012; 13: 187-207.

23. Руководство по эндокринной гинекологии: 3-е изд., стереотипное / под редакцией Е.М. Вихляевой. М.: Медицинское информационное агентство, 2002: 768. Rukovodstvo po endokrinnoy ginekologii [Handbook of Endocrine Gynecology]: $3^{\text {rd }}$ edition, stereotypical. E.M. Vikhlyaeva, ed. Moscow: Meditsinskoe informatsionnoe agentstvo, 2002: 768 (in Russian).

24. Потемкин В.В. Эндокринология. М., 1986: 411. Potemkin V.V. Endokrinologiya [Endocrinology]. Moscow, 1986: 411 (in Russian).

Поступила в редакцию 12.01.2018 Утверждена к печати 2.04.2018

Горобец Людмила Николаевна - д.м.н., профессор, руководитель отделения психонейроэндокринологии. Буланов Вадим Сергеевич - к.м.н., старший научный сотрудник отделения психонейроэндокринологии. Литвинов Александр Викторович, к.м.н., старший научный сотрудник отделения психонейроэндокринологии.

\section{Горобец Людмила Николаевна, gorobetsln@ mail.ru}

УДК 616.895.8:615.03:577.171

For citation: Gorobets L.N., Bulanov V.S., Litvinov A.V. Indices of cortisol and dehydroepiandrosterone sulfate as potential biomarkers of efficiency of antipsychotic therapy in first psychotic episode patients. Siberian Herald of Psychiatry and Addiction Psychiatry. 2018; 2 (99): 127-132. https://doi.org/10.26617/1810-3111-2018-2(99)-127-132

\section{Indices of cortisol and dehydroepiandrosterone sulfate as potential biomarkers of efficiency of antipsychotic therapy in first psychotic episode patients}




\section{Gorobets L.N., Bulanov V.S., Litvinov A.V.}

Moscow Psychiatry Research Institute - Branch of V.P. Serbsky NMRCMH

Poteshnaya Street 3, 107258, Moscow, Russian Federation

\section{ABSTRACT}

Background. The inconsistency of literature data on the effects of antipsychotics of the first and second generations on the levels of prolactin and sex hormones dictates the need for comparative studies of the levels of these hormones at various stages of antipsychotic therapy. Objective. A study of the dynamics of prolactin, estradiol and testosterone levels in serum in patients with paranoid schizophrenia during antipsychotic therapy. Materials and Methods. In 131 patients with paranoid schizophrenia under monotherapy with olanzapine, clozapine, quetiapine and haloperidol, the parameters of prolactin, estradiol and testosterone are studied by the enzyme immunoassay method. Hormonal parameters are identified at baseline (background), by weeks 3-4 and 6-8 of therapy. Results. Haloperidol therapy, regardless of the background values, leads to a significant $(\mathrm{p}<0.01)$ increase in prolactin levels in the blood, and clozapine and quetiapine therapy to a significant $(\mathrm{p}<0.01)$ decrease in the initially elevated prolactin level. The nature of the effect of olanzapine depends on the background level of prolactin: at a prolactin level of more than $1000 \mathrm{mIU} / \mathrm{l}$, the drug leads to a decrease in the level of the hormone; less than $1000 \mathrm{mIU} / \mathrm{l}$ - increases it and does not change the indices of prolactin if they are in the regulatory range. When therapy with antipsychotics is studied, there is a significant $(p<0.01)$ decrease in estradiol levels against a significant $(p<0.01)$ increase in testosterone levels. Conclusion. The authors substantiate the hypothesis that a decrease in the level of estradiol against a background of increased testosterone levels in patients with paranoid schizophrenia promotes the development of menstrual dysfunctions. A key role in increasing testosterone levels in women in the process of antipsychotic therapy is the decrease in the level of estradiol and, to a lesser extent, changes in prolactin levels.

Keywords: schizophrenia, antipsychotic therapy, prolactin, estradiol, testosterone.

Gorobets Lyudmila N., MD, Prof., Head of Psychoneuroendocrinology Department, Moscow Psychiatry Research Institute - Branch of V.P. Serbsky NMRCMH, Moscow, Russian Federation.

Bulanov Vadim S., PhD, senior researcher of Psychoneuroendocrinology Department, Moscow Psychiatry Research Institute - Branch of V.P. Serbsky NMRCMH, Moscow, Russian Federation.

Litvinov Alexander V., PhD, senior researcher of Psychoneuroendocrinology Department, Moscow Psychiatry Research Institute - Branch of V.P. Serbsky NMRCMH, Moscow, Russian Federation.

Gorobets Lyudmila N., gorobetsln@mail.ru 